\title{
In thisissue
}

By John A shkenas, Science Editor

\section{Profiling T-cell gene expression in anergy and arthritis}

(Seearticleon pages519-528)

T cells that losetheir characteristic ability to proliferateand secrete cytokines in responseto antigen stimulation are said to be anergic. Anergy occurs under several different circumstances, suggesting that $T$ cells are poised to become anergic - presumably a protective responsethat hel ps limit immune reactions and prevent responses to self antigens. Still, as Ali and colleagues point out, T cells in at least one autoimmune condition, rheumatoid arthritis (RA), are resistant to apoptosis and antigen stimulation, features that they share with anergic $T$ cell clones. To explore the idea that anergic cells participate in RA pathogenesis, these authors used differential display to identify genes that are induced or suppressed during the onset of anergy, and they tested RA-associated synovial T cells to determine how well they fit the resulting expression profile. Ali et al. identified six transcripts whoseup- or downregulation in RA (as compared with reactivearthritis, a distinct disease, in which T cells remain sensitiveto antigen stimulation) parallels the changes in expression seen in anergic cells. Several of these changes are modest or equivocal, but the suppression of calmodulin expression in RA is consistent and dramatic. Ali et al. further show that cal modulin expression is restored in patients receiving treatment for RA, and they suggest that regulation of cellular cal cium levels represents an important link between the physiology of the anergic cell and the disease-associated T cell in RA.

\section{HIV population shifts following HAART}

(Seearticle on pages 431-438)

Highly activeantiretroviral therapy (HAART) involves combinations of proteaseinhibitors and other drugs to block HIV proliferation. While thereis abundant evidence that this approach reduces viremia, far less is known about its qualitativeeffects on thesurviving HIV population. HIV variants, sometimes called quasispecies, can differ in their host cell tropism, their pathological effects, and the structure of their envelopeglycoprotein, env. Variation in env sequence determines which of two chemokine receptors, CXCR4 or CCR5, will serveas a coreceptor for viral entry. Typically, CCR5-dependent viruses (R5) arethe major population early in thecourse of an infection, and CXCR4dependent viruses (X4) overtakethem later, leading to a drop in CD 4+T-cell count. By following therelativeprevalence of R5 and X4 viruses in a set of women with advanced disease, Philpott et al. now show that R5 viruses can re-emerge as the predominant HIV-1 population following HAART or other combination therapies. Indeed, most of these subjects, even those with little reduction in their overall circulating HIV levels, showed a suppression of X4 strains in preference to $\mathrm{R} 5$ strains. The authors suggest that this qualitative effect could providean additional basisfor the benefit of combination therapies, independent of their quantitative effect on viral titer.

\section{The molecular evolution of viral drug resistance}

(Seearticle on pages 449-455)

As with HIV, the distribution of variants of hepatitis B virus (HBV) can shift in responseto drug therapy. Although viral replication is often controllableusing nucleotideanalogues that inhibit the viral D N A polymerase, drug-resistant variants often emergefollowing chronic treatment, as a result of mutations in thenucleotidebinding site of this enzyme. Here, $\mathrm{O}$ no and colleagues show that known $\mathrm{HBV}$ polymerase point mutants blunt the effect of these drugs but that they do so at the cost of reducing thebaseline replication rate of the virus. H owever, second-sitemutations occurring at one specific residue in the polymerase can restore the catalytic efficiency of the enzyme, generating a virus that is both drug-resistant and quick to replicate. Ono and coworkers also describe the effects of a battery of newly developed antiviral agents on viral replication, comparing wild-typeH BV with several known polymerase point mutants. They confirm that the efficacy of lamivudine, now the standard drug used for controlling HBV, is greatly diminished in all of the drug-resistant viruses studied, but they show that some of the other agents retain significant activity against these variants. Onedrug, the purine analogue entecavir, stands out because of its thousandfold greater potency than lamivudineand its ability to suppress replication of even the most vigorous drug-resistant mutants. For more on the importance of pathogen genotype, see the Perspective series on bacterial polymorphisms, beginning in this issue of the $\mathrm{Cl}$. 